\title{
Erratum: Natural BNS damping of the fast ion instability [Phys. Rev. ST Accel. Beams 2, 044403 (1999)]
}

\author{
D. V. Pestrikov \\ High Energy Accelerator Research Organization (KEK), 1-1 Oho, Tsukuba-shi, Ibaraki-ken 305, Japan \\ (Published 26 November 2001) \\ DOI: 10.1103/PhysRevSTAB.4.119901 \\ PACS numbers: 29.27.-a, 41.85.-p, 99.10.+g
}

Reference [7] should be G. V. Stupakov, T. O. Raubenheimer, and F. Zimmermann, Phys. Rev. E 52, 5499 (1995). The article wrapper (http://prst-ab.aps.org/abstract/PRSTAB/v2/i4/e044403), which is not archival, has been updated. 